included in the study. The proportion of hypothyroidism amongst GCA patients was increased in comparison with controls $(18.2 \%$ vs. $6.91 \%$, respectively, $p$-value $<0.001)$, as was hyperthyroidism $(2.56 \%$ and $1.19 \%$ respectively, $p$ value $<0.001$ ). After controlling for confounders, GCA demonstrated a robust independent association with hypothyroidism on multivariate logistic regression (OR 1.297, 95\% Cl 1.187-1.418, Table 1). In contrast, when a similar model was performed in order to assess the nature of the association between GCA and hyperthyroidism, it was found to be non-significant, with an OR of 1.097

Table 1. Multivariate logistic regression of covariates associated with hypothyroidism

\begin{tabular}{lccc}
\hline Characteristic & OR & $\mathrm{Cl}$ & $\mathrm{P}$ \\
\hline Age & 1.03 & $1.02-1.03$ & $<0.001$ \\
Gender (Female) & 3.32 & $2.93-3.79$ & $<0.001$ \\
BMI & 1.02 & $1.01-1.03$ & $<0.001$ \\
SES: & & & \\
$\quad$ Medium vs. Low & 1.42 & $1.28-1.58$ & $<0.001$ \\
$\quad$ High vs. Low & 1.63 & $1.45-1.83$ & $<0.001$ \\
$\quad$ GCA & 1.30 & $1.12-1.42$ & $<0.001$ \\
\hline
\end{tabular}

BMl: Body Mass Index, kg/m², SES: Socioeconomic status, GCA: Giant Cell Arteritis.

Conclusions: GCA patients have a higher proportion of hypothyroidism in comparison with matched controls. A significant association between GCA and hyperthyroidism was not found. Physicians treating GCA patients should consider screening for thyroid dysfunction on a regular basis.

References:

[1] How J, Bewsher PD, Walker W. Giant-cell arteritis and hypothyroidism. Br Med J. 1977;2(6079):99.

[2] Wiseman P, Stewart K, Rai GS. Hypothyroidism in polymyalgia rheumatica and giant cell arteritis. BMJ. 1989;298(6674):647.

[3] Bowness P, Shotliff K, Middlemiss A, Myles AB. Prevalence of hypothyroidism in patients with polymyalgia rheumatica and giant cell arteritis. Rheumatology. 1991;30(5):349-351.

[4] Duhaut $\mathrm{P}$, Bornet $\mathrm{H}$, Pinède $\mathrm{L}$, et al. Giant cell arteritis and thyroid dysfunction: multicentre case-control study. BMJ. 1999;318(7181):434-435.

[5] Myklebust G, Gran JT. A prospective study of 287 patients with polymyalgia rheumatica and temporal arteritis: clinical and laboratory manifestations at onset of disease and at the time of diagnosis. Rheumatology. 1996;35(11):11611168.

Disclosure of Interest: None declared

DOI: 10.1136/annrheumdis-2017-eular.2555

\section{THU0343 THE EFFECT OF ADALIMUMAB ON CLINICAL MANIFESTATIONS AND PRO-INFLAMMATORY CYTOKINES MILIEU IN PATIENTS WITH BEHCET'S DISEASE}

Y. Braun-Moscovici, Y. Tavor, D. Markovits, K. Toledano, A. Rozin, M.A. Nahir, A. Balbir-Gurman. Rheumatology Department, Rambam Health Care Campus, Rappaport Faculty of Medicine, Technion, Haifa, Israel

Background: Behcet's disease is a multisystemic chronic relapsing inflammatory disease, classified among the vasculitides. The aetiology of Behcet's disease is unknown. Several cytokines, among them TNF- $\alpha$, are involved in the pathogenesis of the disease.

Objectives: We aimed to assess efficacy and safety of Adalimumab (ADA) in patients with active Behcet's arthritis not responding to one or more DMARDS and to assess the impact of treatment on the cytokine milieu.

Methods: Eligible patients (pts) with active arthritis were enrolled in a 24 weeks single center prospective open-label study. Pts who relapsed within 12 weeks following ADA discontinuation could enter a 3 year extension study. The efficacy was assessed by 68 tender and 66 swollen joint count, patient visual analogue scale (VAS) for pain, physician overall disease activity VAS, health assessment questionnaire (HAQ), Behçet's Disease Current Activity Form (BDCAF), C reactive protein (CRP) and erythrocyte sedimentation rate (ESR). TNF- $\alpha$, IL-1 $1 \beta$, IL-6, INF- $\gamma$, $\mathrm{IL}-10$ and IL-17a were evaluated at baseline, after 24 and 48 weeks of treatment, by ProcartaPlex Human High Sensitivity - Immunoassay kit. Trough ADA serum levels and anti-drug antibodies were measured at baseline, week 24 and 48.

Results: Ten pts (6 females),age (mean, standard deviation -SD) 45 (8.4) years, with a disease duration of 11.6 (10) years, were enrolled and treated with subcutaneous ADA $40 \mathrm{mg}$ every 2 weeks for 24 weeks. The results are described

Abstract THU0344 - Table 1 in Table1. A statistically significant improvement was observed in swollen joint count, physician VAS and BDCAF and in IL-6 levels, but not in tender joint count or HAQ. Resolution of oral and urogenital ulcers was achieved in all pts. Significant reduction of pain was reported by $40 \%$ of pts. No relapse of uveitis or other disease manifestations occurred during the study. The reduction in IL-6 levels correlated with the physician VAS and BDCAF but not with HAQ. No correlation was found between change in IL-10 level and VAS pain. The levels of INF- $\gamma$, IL-17A, TNF- $\alpha$ were undetectable in all pts. IL-1 $\beta$ was elevated in 1 patient only. ADA serum trough levels were in the therapeutic range in $7 / 10$ pts. One patient developed high antidrug antibodies titer and ADA serum trough level of 0 with a concomitant increase in VAS pain and IL-6 concentration. Another patient with low ADA trough levels and no antibodies improved after providing ADA weekly. The disease relapsed in 9/10 pts, within 4-6 weeks following ADA interruption, 7 pts enrolled into the extension study.

\begin{tabular}{lccc}
\hline & Baseline mean (SD) & 24 weeks mean (SD) & $\mathrm{P}$ \\
\hline Swollen joints & $4.6(4.2)$ & $0.6(0.4)$ & 0.006 \\
Tender joints & $19(18.7)$ & $12.6(10.9)$ & Non significant (NS) \\
Physician VAS & $51.5(18.5)$ & $24.5(16)$ & 0.002 \\
Patient pain VAS & $72(19)$ & $56(33)$ & $\mathrm{NS}$ \\
BDCAF & $5.4(1.6)$ & $2.1(1.4)$ & 0.001 \\
HAQ & $1.76(0.8)$ & $1.6(0.9)$ & $\mathrm{NS}$ \\
IL-6 pg/ml & $10.06(13.4)$ & $2.02(0.8)$ & 0.042 \\
\hline
\end{tabular}

Conclusions: ADA treatment was well tolerated and achieved a significant improvement in arthritis and mucocutaneous manifestations and lowered IL-6 serum concentration in all study pts but only $40 \%$ reported significant pain reduction. A subset of pts with insufficient improvement in joint tenderness and generalized pain may require comprehensive pain management besides antiinflammatory therapy.

Acknowledgements: ABBVIE donated the study medication and supported the lab work

Disclosure of Interest: None declared

DOI: 10.1136/annrheumdis-2017-eular.4939

\section{THU0344 A RHEUMATOLOGY LED PATHWAY FOR THE INITIAL MANAGEMENT OF GCA IMPROVES DIAGNOSTIC OUTCOMES COMPARED TO THE GENERALIST}

Z. Farah, M. Gulati, M. Matheou, H. Alam, S. Hamdulay. Rheumatology, Northwick Park Hospital, London, United Kingdom

Background: Giant Cell Arteritis (GCA) is a medical emergency requiring prompt and appropriate management to prevent complications. The British Society of Rheumatology (BSR) has set out guidelines on the appropriate management of suspected GCA targeting both primary and secondary-care physicians. Hospitals increasingly use care-pathways to facilitate appropriate initial management of GCA by non-rheumatologists. Evidence is limited, however, on the impact of such interventions on patient care.

Objectives: To evaluate the impact of designing a GCA care-pathway for nonrheumatologists (acute and general physicians) on patient care in terms of: (i) duration from referral to temporal artery biopsy (TAB) and rheumatology review; (ii) glucocorticoid therapy burden; and (iii) proportion of referrals with a final diagnosis of GCA

Methods: We performed a retrospective study of all patients diagnosed with GCA between 3 periods: prior to introducing a GCA pathway (2007-2009) rheumatology led GCA pathway (2010-2012), and non-rheumatology led GCA pathway (2012-2016). We identified patients from a TAB database and collected general demographic data, initiation of glucocorticoid therapy, referral for TAB and rheumatology clinic, date of TAB and clinic review, biopsy findings, and final diagnosis.

Results: Table 1 summarises the main findings. After introducing the rheumatology-led pathway (2010-2012), rate of referrals for TAB per month declined to 0.78 , the proportion of patients having a TAB within 14 days of referral reached $100 \%$, and the proportion of patients with a positive biopsy increased to $30 \%$ suggesting appropriate use of the pathway and an improvement in care. However introducing a non-rheumatology led GCA pathway (2012-2016), led to increased referral numbers. The proportion of TAB within 14 days decreased, and the proportion with a positive biopsy declined (17\%).

\begin{tabular}{|c|c|c|c|}
\hline & Before GCA Pathway & Rheumatology Led GCA Pathway (1st Cycle) & Non-Rheumatology Led GCA Pathway (2nd Cycle) \\
\hline Dates & Pre-2010 (Jan 07-Sep 09) & 2010 to 2012 (Jan 10-Mar 12) & 2016 (Nov 12-June 16) \\
\hline Study Design & Retrospective & Retrospective & Retrospective \\
\hline Source of Data & Histology database, electronic records & Histology database, electronic records & Histology database, electronic records \\
\hline Number of Patients Biopsied & 54 & 21 & 129 \\
\hline Number of Months & 33 & 27 & 44 \\
\hline Rate of Biopsies (pts/month) & 1.64 & 0.78 & 2.93 \\
\hline Days from referral to TAB; Median (min-max) & $5(0-56)$ & $5(0-13)$ & $9(0-45)$ \\
\hline Proportion with TAB within 14 days & $87 \%$ & $100 \%$ & $66.2 \%$ \\
\hline Proportion with a positive biopsy for GCA & $26 \%$ & $30 \%$ & $17 \%$ \\
\hline Mean Age (years) & 73 & 73 & 71 \\
\hline Gender Ratio (M:F) & $18: 36$ (F 66.7\%) & $4: 17$ (F 80.9\%) & $48: 81(\mathrm{~F} 62.7 \%)$ \\
\hline OUTCOME & GCA Pathway introduced & Pathway continued & Pathway review \\
\hline
\end{tabular}


The non-rheumatology led GCA pathway was associated with higher glucocorticoid burden. In this cohort, $23 / 55(41.8 \%)$ patients who were found not to have GCA received more than 21 days of high dose steroids (40-60mg Prednisolone) whilst awaiting rheumatology review. 4 patients whose final diagnosis was not GCA received high dose steroids for more than 30 days ( $\max 109$ days).

Conclusions: 1. Availability of a rheumatology-led GCA pathway leads to improved care for patients with suspected GCA

2. Easy access for referral of patients with headache as assessed by the non-specialist can lead to over-use of the pathway and inappropriate referrals

3. Non-rheumatology-led GCA pathway can lead to a high glucocorticoid burden, especially in an elderly demographic with other comorbidities

Disclosure of Interest: None declared

DOI: 10.1136/annrheumdis-2017-eular.1248

\section{THURSDAY, 15 JUNE 2017 Spondyloarthritis - treatment}

\section{THU0345 REAL-LIFE EFFECTIVENESS OF TNF INHIBITORS IN AXIAL SPONDYLARTHRITIS: ARE CHANGING NATIONAL POLICIES ON CHOICE OF TNF INHIBITOR REFLECTED IN RESPONSE TO TREATMENT?}

E.K. Kristianslund ${ }^{1}$, K.M. Fagerli ${ }^{1}$, E. Lie ${ }^{1}$, A. Wierød ${ }^{2}$, S. Kalstad ${ }^{3}$, E. Rødevand ${ }^{4}$, F. Krøll ${ }^{5}$, P. Mielnik ${ }^{6}$, T.K. Kvien ${ }^{1}$, I.C. Olsen ${ }^{1} .{ }^{1}$ Department of Rheumatology, Diakonhjemmet Hospital, Oslo; ${ }^{2}$ Department of Rheumatology, Drammen Hospital, Drammen; ${ }^{3}$ Department of Rheumatology, University Hospital of North Norway, Tromsø; ${ }^{4}$ Department of Rheumatology, St. Olavs Hospital, Trondheim; ${ }^{5}$ Revmatismesykehuset, Lillehammer; ${ }^{6}$ Department of Rheumatology, Førde Central Hospital, Førde, Norway

Background: Tumour necrosis factor inhibitors (TNFi) have revolutionized treatment of axial spondylarthritis (axSpa). The five different available TNFi have not been compared directly, and whether effectiveness differs between agents is unknown. In Norway national authorities consider the different TNFi equivalent, and since 2009 the least expensive drug following an annual national tender has been the drug-of-choice in the publicly funded healthcare system. This has lead to variations across different years in drug use where choice of TNFi has been predominantly based on national price policy and not clinical characteristics.

Objectives: Comparing response to TNFi during the first year of treatment of axSpA in biologics-naïve patients over years with highly varying uptake of different TNFi.

Methods: We included the 981 biologics-naivve patients with axSpA from the NOR-DMARD register who started their first TNFi from 2009 through 2015. The preferred drugs in national recommendations were: 2009 adalimumab, 2010 golimumab, 2011 etanercept, 2012 etanercept, 2013 golimumab, 2014 certolizumab, 2015 certolizumab/biosimilar infliximab (CT-P13). We compared the estimated change in Ankylosing Spondylitis Disease Activity Score (ASDAS) between treatment years at 3,6 and 12 months after treatment start using a mixed model with subject-specific random intercept, adjusting for baseline disease activity, age, sex and treatment centre.

Results: Demographics, drug uptake and baseline characteristics for each year 2009-2015 are listed in table 1. The preferred drug was started in $57-91 \%$ of patients. There was a trend towards lower ASDAS and disease duration over time. There were no differences in treatment effectiveness between the years, regardless of the substantial differences in type of TNFi used (figure).

Conclusions: Real-life data do not show differences in response to TNFi despite large annual variation in type of TNFi prescribed, indicating similar effectiveness of the available TNFi in patients with axSpA. This supports the practice of selecting drug based on cost and feasibility of use, as is the current practice in Norway. Further adoption of this principle can provide access to TNFi treatment to more patients, as it reduces costs and healthcare resources are limited.

Disclosure of Interest: E. K. Kristianslund: None declared, K. M. Fagerli: None declared, E. Lie Consultant for: AbbVie, Celgene, Hospira, Pfizer, UCB, A. Wierød: None declared, S. Kalstad: None declared, E. Rødevand: None declared, F. Krøll: None declared, P. Mielnik: None declared, T. K. Kvien Consultant for: AbbVie, Biogen, BMS, Boehringer Ingelheim, Celltrion, Eli Lilly, Epirus, Janssen, Merck-Serono, MSD, Mundipharma, Novartis, Oktal, Orion Pharma, Hospira/Pfizer, Roche, Sandoz, UCB, I. C. Olsen: None declared

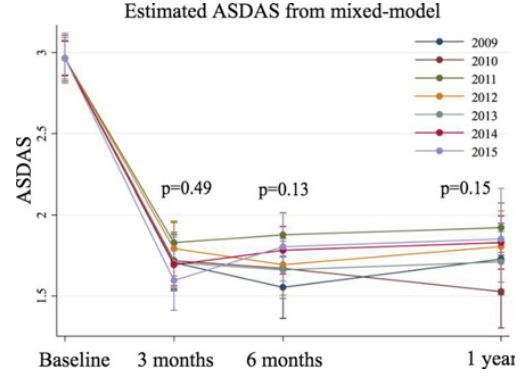

DOI: 10.1136/annrheumdis-2017-eular.2161

\section{THU0346 CONCOMITANT FIBROMYALGIA IN AXIAL SPONDYLOARTHRITIS HAS A NEGATIVE IMPACT ON TNF ALPHA BLOCKERS TREATMENT EFFECT IN REAL LIFE}

A. Moltó, A. Etcheto, L. Gossec, S. Perrot, N. Boudersa, P. Claudepierre, N. Roux, F. Berenbaum, A. Martin, L. Sparsa, P. Coquerelle, M. Soubrier, M. Dougados. Predict-SpA Study Group, Paris, France

Background: Coexisting fibromyalgia (FM) in axial spondyloarthritis (axSpA) can represent therapeutic challenges, particularly when evaluating the treatment effect of biologics (i.e. TNF alpha blockers (TNFb)). Indeed, since FM patients often report high levels of pain and disability there is the risk of classifying such patients as refractory to TNFb (ie, as not reaching a significant improvement in disease activity).

Objectives: To evaluate the impact of concomitant FM on the TNFb treatment effect in axSpA

Methods: Design: Prospective observational national study with 2 visits 3 months apart (baseline and 12 weeks after TNFb initiation) (Predict-SpA study ClinicalTri als.gov: NCT03039088). Patients: axSpA patients (diagnosis according to treating rheumatologist) initiating a TNFb. Data collection: the FiRST questionnaire (Fibromyalgia Rapid Screen Test) which screens for FM, patients and disease characteristics and effectiveness measures (e.g. ASAS response components). Statistical analysis: FM positive screening was defined by a FIRST score $\geq 5 / 6$; the primary efficacy outcome was the ASAS 40. Non-responder imputation and baseline observation carried forward imputation (for binary and continuous outcome variables, respectively) was performed. Impact of FM on the TNFb treatment effect was evaluated by multivariable logistic regression, with ASAS 40 as the dependent variable and FM as the independent variable; were also included in the model other factors previously reported in the literature as associated with treatment efficacy (i.e. X-ray and MRI sacroiliitis, abnormal CRP (>6mg/L), HLA B-27, smoking status, previous TNFb exposure, age $<40$ and male gender).

Results: Among the 527 patients enrolled in the study, 508 patients were analysed. Mean age was $41.4( \pm 11.6), 237(46.7 \%)$ were women, with a $6.1 \pm 8.5$ mean disease duration. Among them, 192 (37.8\%) were screened as FM by the FiRST questionnaire. Overall efficacy of the TNFb was good (ASAS40, 201/508 $(39.6 \%))$ though 50 patients $(9.8 \%)$ patients discontinued the TNFb before the follow-up visit and were considered as non-responders.

Patients with FM presented less frequently an ASAS 40 response (87/192 $(45.3 \%)$ vs $171 / 316(54.1 \%)$, for the FM vs non-FM groups according to the FIRST definition. Presence of FM was independently associated with poorer ASAS40 response (adjusted odds ratio, OR $=0.5[95 \% \mathrm{Cl} 0.3-0.8]$ ) while X-ray sacroiliitis (1.8 [1.2 - 2.8]), abnormal CRP (1.6 [1.0 - 2.4]) and absence of previous exposure to TNFb $(1.7[1.1-2.6])$ were found to be associated with an ASAS40 response.

Conclusions: This study 1) confirms the "conventional" predisposing factors of TNFb treatment response such as X-ray sacroiliitis, abnormal CRP and absence of previous exposure to TNFb; and 2) suggests that concomitant FM influences treatment response. FM deserves to be screened in axSpA, in particular in case of a decision to initiate a TNFb therapy.

References:

[1] Perrot S, Bouhassira D, Fermanian J; Cercle d'Etude de la Douleur en Rhumatologie. Development and validation of the Fibromyalgia Rapid Screening Tool (FiRST). Pain. 2010;150:250-6.

Abstract THU0345 - Table 1. Demographics and baseline characteristics

\begin{tabular}{|c|c|c|c|c|c|c|c|c|}
\hline & 2009 & 2010 & 2011 & 2012 & 2013 & 2014 & 2015 & $\mathrm{p}$-value \\
\hline $\mathrm{N}$ & 110 & 104 & 200 & 124 & 148 & 192 & 103 & \\
\hline Age (years), mean (SD) & $42.3(11.2)$ & $40.3(11.5)$ & $41.7(13.0)$ & $40.0(12.0)$ & $40.9(12.7)$ & $41.6(11.8)$ & $41.6(12.3)$ & 0.76 \\
\hline Proportion male & $66.1 \%$ & $61.2 \%$ & $50.8 \%$ & $55.6 \%$ & $52.7 \%$ & $54.7 \%$ & $57.3 \%$ & 0.19 \\
\hline Years since diagnosis, median (IQR) & $4.51(0.77,14.69)$ & $3.79(0.61,12.74)$ & $2.63(0.47,12.88)$ & $1.70(0.56,11.08)$ & $0.76(0.22,6.78)$ & $0.60(0.19,3.44)$ & $1.41(0.18,10.76)$ & $<0.001$ \\
\hline ASDAS, mean (SD) & $3.26(0.84)$ & $3.20(0.92)$ & $3.10(0.94)$ & $2.96(1.01)$ & $3.02(0.87)$ & $2.87(0.89)$ & $2.71(1.01)$ & $<0.001$ \\
\hline Adalimumab & $90.9 \%$ & $29.8 \%$ & $22.0 \%$ & $14.5 \%$ & $24.3 \%$ & $12.0 \%$ & $0.0 \%$ & $<0.001$ \\
\hline Certolizumab & $0.0 \%$ & $1.9 \%$ & $0.0 \%$ & $0.8 \%$ & $12.8 \%$ & $77.1 \%$ & $49.5 \%$ & \\
\hline Etanercept & $7.3 \%$ & $8.7 \%$ & $63.5 \%$ & $69.4 \%$ & $4.7 \%$ & $2.6 \%$ & $1.9 \%$ & \\
\hline Golimumab & $0.0 \%$ & $58.7 \%$ & $11.0 \%$ & $11.3 \%$ & $57.4 \%$ & $2.6 \%$ & $4.9 \%$ & \\
\hline Infliximab & $1.8 \%$ & $1.0 \%$ & $3.5 \%$ & $4.0 \%$ & $0.7 \%$ & $0.5 \%$ & $0.0 \%$ & \\
\hline Biosimilar infliximab & $0.0 \%$ & $0.0 \%$ & $0.0 \%$ & $0.0 \%$ & $0.0 \%$ & $5.2 \%$ & $43.7 \%$ & \\
\hline
\end{tabular}

p-values for between-year differences. 